\title{
Human recombinant follicle stimulating hormone (rFSH) compared to urinary human menopausal gonadotropin (HMG) for ovarian stimulation in assisted reproduction: a literature review and cost evaluation
}

\author{
P. E. Levi Setti · C. Alviggi • G. L. Colombo • \\ C. Pisanelli $\cdot$ C. Ripellino $\cdot$ S. Longobardi $\cdot$ \\ P. L. Canonico · G. De Placido
}

Received: 21 July 2014 / Accepted: 21 October 2014 / Published online: 6 December 2014

(c) The Author(s) 2014. This article is published with open access at Springerlink.com

\begin{abstract}
Background Gonadotropins are protein hormones which are central to the complex endocrine system that regulates normal growth, sexual development, and reproductive function. There is still a lively debate on which type of gonadotropin medication should be used, either human menopausal gonadotropin or recombinant follicle-stimulating hormone. The objective of the study was to perform a systematic review of the recent literature to compare recombinant follicle-stimulating hormone to human menopausal gonadotropin with the aim to assess any differences in terms of efficacy and to provide a cost evaluation based on findings of this systematic review.

Methods The review was conducted selecting prospective, randomized, controlled trials comparing the two gonadotropin medications from a literature search of several databases. The outcome measure used to evaluate efficacy
\end{abstract}

\section{P. E. Levi Setti}

Humanitas Fertility Center, Division of Gynaecology

and Reproductive Medicine, Department of Gynaecology,

Humanitas Research Hospital, Rozzano, Milan, Italy

\section{Alviggi}

Dipartimento Universitario di Neuroscienze, Scienze

Riproduttive ed Odontostomatologiche, Università degli Studi di

Napoli, "Federico II", Naples, Italy

\section{G. L. Colombo}

Department of Drug Sciences, University of Pavia, Pavia, Italy

G. L. Colombo

S.A.V.E. Studi Analisi Valutazioni Economiche, Milan, Italy

C. Pisanelli

Hospital Pharmacist, ACO San Filippo Neri, Rome, Italy was the number of oocytes retrieved per cycle. In addition, a cost evaluation was performed based on retrieved efficacy data.

Results The number of oocytes retrieved appeared to be higher for human menopausal gonadotropin in only 2 studies while 10 out of 13 studies showed a higher mean number of oocytes retrieved per cycle for recombinant folliclestimulating hormone. The results of the cost evaluation provided a similar cost per oocyte for both hormones.

Conclusions Recombinant follicle-stimulating hormone treatment resulted in a higher oocytes yield per cycle than human menopausal gonadotropin at similar cost per oocyte.

Keywords FSH $\cdot \mathrm{HMG} \cdot$ Human menopausal gonadotropin - Recombinant follicle stimulating hormone · Systematic review · Cost evaluation · Gonadotropins . Infertility $\cdot$ Assisted reproduction

\author{
C. Pisanelli \\ Società Italiana Di Farmacia Ospedaliera, Milan, Italy \\ C. Ripellino ( $\square)$ \\ CSD Medical Research Srl, Viale Jenner n 53, 20159 Milan, Italy \\ e-mail: claudio.ripellino@cegedim.com \\ S. Longobardi \\ Medical Department, MerckSerono S.p.A, Rome, Italy \\ P. L. Canonico \\ Dipartimento di Scienze del Farmaco, Università del Piemonte \\ Orientale, Largo Donegani 2, Novara, Italy \\ G. De Placido \\ University Department of Obstetrics, Gynaecology, Urology \\ and Reproductive Medicine, University of Naples Federico II, \\ Naples, Italy
}




\section{Introduction}

Gonadotropins are protein hormones secreted by gonadotrope cells of the anterior pituitary of vertebrates [1], which are central to the complex endocrine system that regulates normal growth, sexual development, and reproductive function. The two key hormones, follicle-stimulating hormone (FSH) and luteinizing hormone (LH), act synergistically in reproduction, stimulating the growth and recruitment of immature ovarian follicles in the ovary and primary spermatocytes in the testis to undergo the first division of meiosis and to form secondary spermatocytes, in women and men, respectively.

Gonadotropin treatments can be used to stimulate ovulation in women with low natural gonadotropin or estrogen levels, when clomiphene treatment has been ineffective in regulating ovulation caused by polycystic ovary syndrome, for developing multiple egg follicles in the ovaries (retrieved and used in assisted reproductive techniques), in combination with intrauterine insemination for couples with unexplained infertility when clomiphene was not effective. In men, gonadotropin therapy can improve low sperm counts caused by low levels of natural gonadotropins.

For this reason, gonadotropin medications have been the cornerstone of infertility treatment since 1950, when human menopausal gonadotropin (HMG) was first introduced into clinical practice [2], but clinical trials started only in the 1960s [3, 4]. A first alternative medication to $\mathrm{HMG}$, which contained an equal ratio of $\mathrm{FSH}$ and $\mathrm{LH}$, became available in the late 1960s; following different purification processes, urinary FSH (uFSH) was still urinederived, but largely purified of LH [5]. The final product contained $150 \mathrm{IU}$ of FSH and $1 \mathrm{IU}$ of $\mathrm{LH}$ per milligram of protein (though not of co-purified proteins). Further technological advances made it possible to obtain uFSH with even less amount of LH, and in the 1990s highly purified FSH (HP-FSH), which contains $<0.1$ IU of LH activity and $<5 \%$ of unidentified urinary proteins, and highly purified HMG (HP-HMG), with the same labeled ratio of FSH: LH activity of HMG, became available [6, 7].

In the late 1990's, a different type of gonadotropin had been developed: using recombinant DNA technology, recombinant FSH (rFSH) was produced, obtaining preparations that have high purity and biological potency and are completely LH free $[8,9]$.

Following commercialization of recombinant $\mathrm{FSH}$, there has been much controversy with regard to the type of gonadotropin which should be utilized. The present manuscript presents a systematic review of the literature comparing rFSH and HMG with the aim of determining differences in efficacy between these two compounds, as well as, a cost evaluation conducted from the findings of the review.

\section{Materials and methods}

The analysis in this article is based on previously conducted studies, and does not involve any new studies of human or animal subjects performed by any of the authors.

Identification of literature

To assess the efficacy of urinary HMG and rFSH therapies, a literature search of the National Library of Medicine and the National Institutes of Health (PubMed), Medline and Cochrane Controlled Trials Register (i.e., CENTRAL, The Cochrane Library) electronic databases was performed using the following keywords: 'HMG', 'human menopausal gonadotropin', 'recombinant follicle stimulating hormone' and 'recombinant FSH'. Only prospective, randomized, controlled trials comparing recombinant $\mathrm{FSH}$ versus HMG treatments with an adequate sample size were included, assuming a population of at least 15 women by arms to avoid potential bias due to considering small studies. No additional selection on patients characteristics, indications, treatment protocols were applied. Studies selected for inclusion in the review were identified by two experienced health economists (C. Ripellino and A. Guasconi); the reference lists of review articles and included studies drafted by each researcher were compared in order to set the final eligible studies list. No attempts were made to contact authors for additional information.

Study selection and outcome

The outcome measure used to evaluate treatment efficacy was the number of oocytes retrieved per cycle.

This outcome was chosen since the number of oocytes retrieved is directly associated with the stimulating effect of gonadotropins, while other outcomes, such as live birth rate, depend not only on gonadotropins but also on many other interventions and factors (e.g., male factor, quality of laboratory), making it difficult to create a direct cause-effect relation between ovarian stimulation and live birth rate.

Moreover, some studies investigated the association between egg number and live birth rate following in vitro fertilization treatments and suggested that the number of eggs is a robust surrogate parameter for clinical success $[10,11]$.

A total of 59 articles were found (Fig. 1). Subsequently, 46 articles were excluded for the following reasons: urinary FSH versus recombinant FSH treatment $(n=36)$, duplicate publications $(n=2)$, combined analysis using two previous trials $(n=1)$, abstract availability only $(n=1)$, no oocytes outcome $(n=4)$, very small trial (less than 30 patients, $n=2)$. The remaining 13 studies were considered for this publication [12-24] (Table 1). 
Fig. 1 Identification and selection of the studies to be included

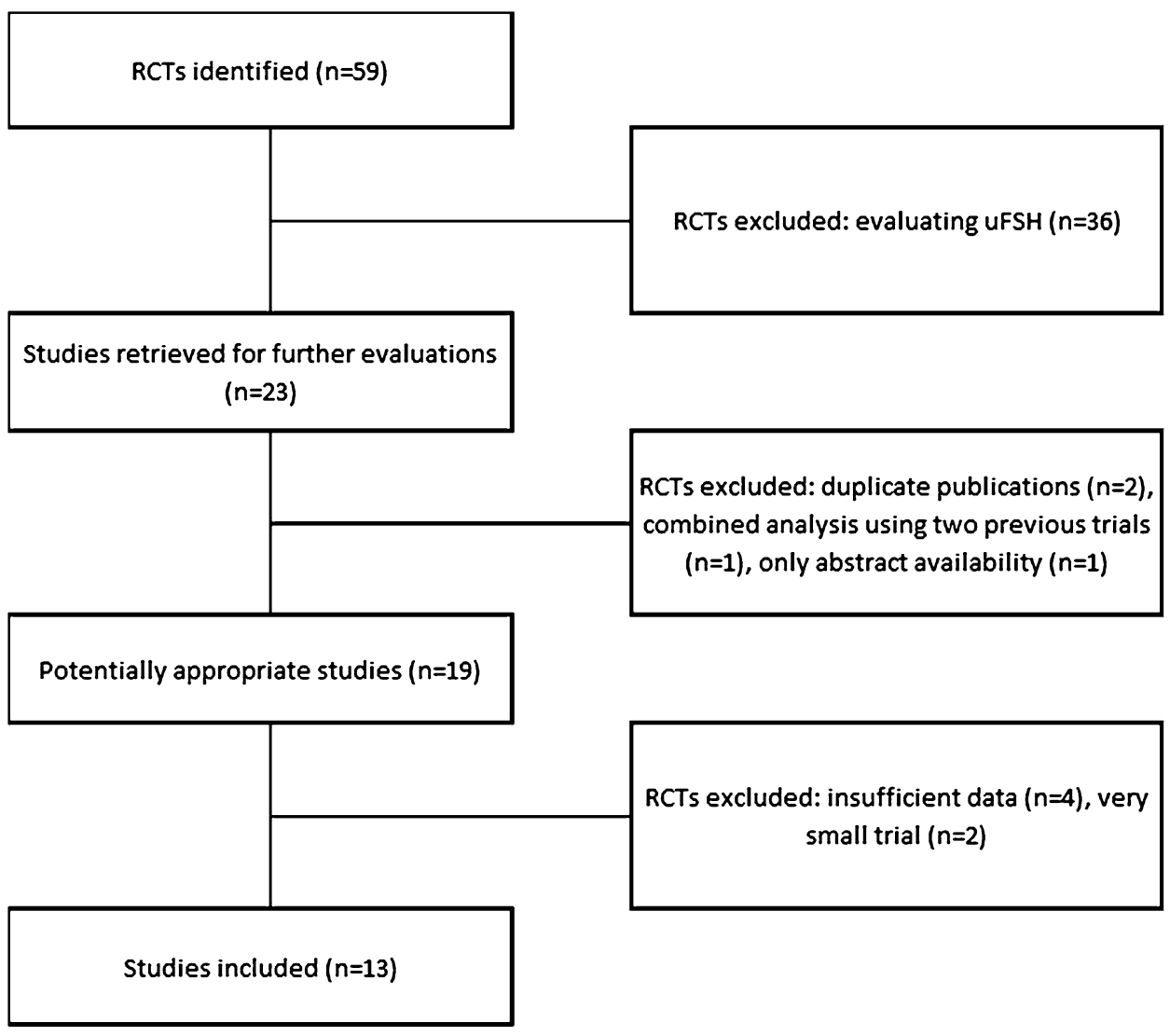

Legends: RCT: randomized clinical trial, uFSH: urinary follicle-stimulating hormone

\section{Cost analysis}

Cost calculations were performed using Italian treatment costs and findings from literature review. Starting from individual studies total dose (IU) and number of oocytes retrieved per cycle, then applying Italian gonadotropin prices, it was possible to obtain the cost per oocyte for each study.

Unit costs used were obtained from the price database available on the Codifa Database [25], last updated in January 2014 , i.e. $€ 38.58$ per vial of $\mathrm{rFSH}$, and $€ 26.57$ per vial of HMG. Only gonadotropin costs were considered, assuming the cost of other resources to be identical or captured by treatment charges that do not differentiate between stimulation protocols.

\section{Results}

Efficacy

About half of the included studies found that rFSH was associated with a lower mean total dose in comparison with HMG (Westergaard et al. [16], Rashidi et al. [19], Andersen et al. [20], Hompes et al. [22], Devroey et al. [23] and Ye et al. [24]), while in other publications (Jansen et al. [12], Gordon et al. [13], NG et al. [14], Strehler et al. [15], Balash et al. [17], Kilani et al. [18] and Bosch et al. [21]) HMG was associated with a lower total mean dose (Table 2).

The mean total dose ranged from 1353 IU to 2624 IU for $\mathrm{rFSH}$ and from $1365 \mathrm{IU}$ to $2508 \mathrm{IU}$ for HMG. The main outcome, i.e., the number of oocytes retrieved, was observed to be higher for HMG in 2 studies only (Kilani et al. [18] and Rashidi et al. [19]); 10 out of 13 studies showed a higher mean number of oocytes for $\mathrm{rFSH}$, while Westergaard et al. [16] found the same mean value for both rFSH and HMG. The mean number of oocytes retrieved ranges from 6.8 to 14.4 for $\mathrm{rFSH}$ and from 7.2 to 12.9 for HMG (Table 2).

\section{Costs}

Results of the economic evaluation are presented in Table 3. The ovarian stimulation with rFSH compared to HMG generated a cost per oocyte that varies from $€ 65$ to $€ 153$ for recombinant therapy and from $€ 55$ to $€ 109$ for urinary therapy; thus, the difference between therapies ranges from $-€ 0.1$ to $€ 77$. 
Table 1 Characteristics of included studies

\begin{tabular}{|c|c|c|}
\hline & Population & Interventions \\
\hline Jansen et al. [12] & 109 Women undergoing IVF & $\begin{array}{l}\text { rFSH vs HMG at a starting dose of } 150 \mathrm{IU} \text { for rFSH and } \\
225 \mathrm{IU} \text { for HMG }\end{array}$ \\
\hline Gordon et al. [13] & 128 Women undergoing IVF & $\begin{array}{l}\text { rFSH vs HMG at a starting dose of } 225 \mathrm{IU} \text { in a long luteal } \\
\text { GnRHa protocol }\end{array}$ \\
\hline NG et al. [14] & 40 Women undergoing ICSI & $\begin{array}{l}\text { rFSH vs HMG at a starting dose of } 300 \text { IU for } 2 \text { days, then } \\
150 \mathrm{IU}\end{array}$ \\
\hline Strehler et al. [15] & 578 Women undergoing IVF or ICSI & rFSH vs HMG at a starting dose from 150 to $450 \mathrm{IU}$ \\
\hline Westergaard et al. [16] & 379 Women undergoing IVF & $\begin{array}{l}\text { rFSH vs HMG at a starting dose of } 225 \mathrm{IU} \text { in a long luteal } \\
\text { GnRHa protocol }\end{array}$ \\
\hline Balash et al. [17] & $\begin{array}{l}60 \text { Patients undergoing ICSI and having unexplained or } \\
\text { male-related primary infertility }\end{array}$ & $\begin{array}{l}\text { rFSH vs HMG at a starting dose of } 150 \text { IU in a long luteal } \\
\text { GnRHa protocol }\end{array}$ \\
\hline Kilani et al. [18] & 100 Women undergoing IVF & $\begin{array}{l}\text { rFSH vs hp-HMG at a starting dose of } 150 \mathrm{IU} \text { in a GnRHa } \\
\text { protocol }\end{array}$ \\
\hline Rashidi et al. [19] & 60 Women undergoing ICSI & rFSH vs HMG at a starting dose of $150 \mathrm{IU}$ \\
\hline Andersen et al. [20] & 731 Infertile women undergoing IVF & $\begin{array}{l}\text { rFSH vs hp-HMG at a starting dose of } 225 \mathrm{IU} \text { in a GnRH- } \\
\text { antagonist protocol }\end{array}$ \\
\hline Bosch et al. [21] & 280 Infertile women undergoing IVF or ICSI & $\begin{array}{l}\text { rFSH vs hp-HMG at a starting dose of } 225 \mathrm{IU} \text { in a fixed } \\
\text { GnRH-antagonist protocol }\end{array}$ \\
\hline Hompes et al. [22] & 629 Infertile women undergoing IVF & $\begin{array}{l}\text { rFSH vs hp-HMG at a starting dose of } 150 \mathrm{IU} \text { in a GnRH-a } \\
\text { long protocol }\end{array}$ \\
\hline Devroey et al. [23] & 749 Infertile patients undergoing ICSI & $\begin{array}{l}\text { rFSH vs hp-HMG at a starting dose of } 150 \mathrm{IU} \text { in a GnRH- } \\
\text { antagonist protocol }\end{array}$ \\
\hline Ye et al. [24] & 127 Infertile women undergoing IVF or ICSI & rFSH vs hp-HMG at a starting dose of $225 \mathrm{IU}$ \\
\hline
\end{tabular}

$I V F$ in vitro fertilization, ICSI intracytoplasmic sperm injection, $r F S H$ recombinant follicle stimulating hormone, $h p-H M G$ highly purified human menopausal gonadotropin, $I U$ international units, $G n R H$ gonadotropin-releasing hormone, $G n R H$ - $a$ gonadotropin-releasing hormone agonist

Table 2 Table of outcome measures

\begin{tabular}{|c|c|c|c|c|}
\hline & \multicolumn{2}{|l|}{ Total dose (IU) } & \multicolumn{2}{|c|}{ No. of retrieved oocytes } \\
\hline & $\mathrm{rFSH}($ means \pm std) & HMG (means \pm std) & $\mathrm{rFSH}($ means \pm std) & HMG (means \pm std) \\
\hline Jansen et al. [12] & $1,410 \pm 228$ & $1,365 \pm 228$ & $11.2 \pm 6.8$ & $8.3 \pm 6.2$ \\
\hline Gordon et al. [13] & $2,025 \pm 350$ & $1,981 \pm 570$ & $12 \pm 6$ & $10 \pm 7$ \\
\hline NG et al. [14] & $1,800 \pm 270$ & $1,650 \pm 270$ & $12.6 \pm 8.9$ & $9.6 \pm 8.1$ \\
\hline Strehler et al. [15] & $2,150 \pm 797$ & $1,516 \pm 545$ & $12.29 \pm 7.8$ & $9.67 \pm 5.92$ \\
\hline Westergaard et al. [16] & $2,242 \pm 375$ & $2,280 \pm 435$ & $12.9 \pm 6.8$ & $12.9 \pm 6.7$ \\
\hline Balash et al. [17] & $2,449 \pm 885$ & $1,922 \pm 379$ & $11.79 \pm 4.55$ & $9.1 \pm 4.35$ \\
\hline Kilani et al. [18] & $2,025 \pm 795$ & $1,680 \pm 530$ & $6.8 \pm 3.9$ & $7.9 \pm 4.6$ \\
\hline Rashidi et al. [19] & $2,138 \pm 800$ & $2,250 \pm 800$ & $8.7 \pm 8.5$ & $9 \pm 6.2$ \\
\hline Andersen et al. [20] & $2,385 \pm 622$ & $2,508 \pm 729$ & $11.8 \pm 5.7$ & $10.0 \pm 5.4$ \\
\hline Bosch et al. [21] & $2,624 \pm 801$ & $2,481 \pm 994$ & $14.4 \pm 8.1$ & $11.3 \pm 6.0$ \\
\hline Hompes et al. [22] & $1,759.7$ & $1,821.0$ & 10.56 & 7.76 \\
\hline Devroey et al. [23] & $1,353 \pm 296$ & $1,433 \pm 371$ & $10.7 \pm 5.8$ & $9.1 \pm 5.2$ \\
\hline Ye et al. [24] & $2,162.7 \pm 399.4$ & $2,219 \pm 502.7$ & $10.2 \pm 5.2$ & $7.2 \pm 4.2$ \\
\hline
\end{tabular}

A relevant difference in the cost per oocyte between rFSH and HMG has been observed in Kilani et al. (€ 77) [18]; this cost difference is greater than the values of the other studies, which varied from $-€ 0.1$ to $€ 37$.

\section{Discussion}

This review evaluated efficacy in terms of number of oocytes retrieved per cycle and the costs, calculated 
Table 3 Table of costs

\begin{tabular}{llll}
\hline & \multicolumn{2}{l}{ Cost per oocyte } \\
\cline { 2 - 4 } & $\mathrm{rFSH}$ & HMG & Difference in costs \\
\hline Jansen et al. [12] & $€ 64.8$ & $€ 58.3$ & $€ 6.5$ \\
Gordon et al. [13] & $€ 86.8$ & $€ 70.2$ & $€ 16.6$ \\
NG et al. [14] & $€ 73.5$ & $€ 60.9$ & $€ 12.6$ \\
Strehler et al. [15] & $€ 90.0$ & $€ 55.5$ & $€ 34.4$ \\
Westergaard et al. [16] & $€ 89.4$ & $€ 62.6$ & $€ 26.8$ \\
Balash et al. [17] & $€ 106.9$ & $€ 74.8$ & $€ 32.0$ \\
Kilani et al. [18] & $€ 153.2$ & $€ 75.3$ & $€ 77.8$ \\
Rashidi et al. [19] & $€ 126.4$ & $€ 88.6$ & $€ 37.8$ \\
Andersen et al. [20] & $€ 104.0$ & $€ 88.9$ & $€ 15.1$ \\
Bosch et al. [21] & $€ 93.7$ & $€ 77.8$ & $€ 16.0$ \\
Hompes et al. [22] & $€ 85.7$ & $€ 83.1$ & $€ 2.6$ \\
Devroey et al. [23] & $€ 65.0$ & $€ 55.8$ & $€ 9.3$ \\
Ye et al. [24] & $€ 109.1$ & $€ 109.2$ & $-€ 0.1$ \\
\hline
\end{tabular}

applying Italian treatment costs to the findings of the retrieved studies, of rFSH and HMG in ovarian stimulation protocols in infertile women. The results of this systematic review suggest that $\mathrm{rFSH}$ is likely to be more effective than HMG. The number of oocytes retrieved per cycle was higher in almost all studies considered, with a similar total dose used for both rFSH and HMG.

The results of the economic evaluation provided a similar cost per oocyte for rFSH and HMG (with maximum cost differences of $€ 37$ for Rashidi et al. [19] and $€ 77$ for Kilani et al. [18]).

The present study findings are in agreement with the results of the largest meta-analyses published to date on this subject [26-29].

The Cochrane review by van Wely et al. [26] comparing rFSH to other gonadotropins irrespective of the downregulation protocol used, presented evidence of a major oocyte production for $\mathrm{rFSH}$ in comparison with $\mathrm{HMG}$ in most of the considered studies.

In the 5 trials included in the meta-analysis conducted by Jee et al. [27], more oocytes were retrieved in the rFSH group, with the exception of the trial by Kilani et al. [18]. In the meta-analysis by Lehert et al. [28], treatment with human menopausal gonadotropins resulted in fewer oocytes (mean difference $-1.54 ; 95 \% \mathrm{CI}-2.53$ to -0.56 ; $p<0.0001)$ compared to rFSH. Furthermore, a higher total dose of human menopausal gonadotropin was necessary [mean difference 235.46 IU (95 \% CI 16.62-454.30; $p=0.03)]$.

A meta-analysis conducted by Wex et al. [29] showed a greater number of oocytes with $\mathrm{rFSH}$ (mean difference 1.96; $95 \%$ CI 1.02-2.90). Furthermore, authors developed a cost-minimization model where $\mathrm{rFSH}$ has been found to be cost-saving, at $90,195 \mathrm{kr}(€ 10,282$ or $\$ 13,394)$ with rhFSH compared to $96,436 \mathrm{kr}(€ 10,994$ or $\$ 14,321)$ with HP-HMG per live-birth.

A retrospective databases chart review from 4 European countries investigated gonadotropins usage, oocyte and embryo yield, and pregnancy outcomes in IVF cycles using rFSH or HP-HMG have been conducted by Trew et al. [30]. The group demonstrated a significantly lower drug usage per cycle for rFSH than HP-HMG (22.6\% higher for HP-HMG; $p<0.01$ ) and a significantly greater average oocyte yield per IVF cycle in patients treated with $\mathrm{rFSH}$ in comparison with HP-HMG $(10.80 \pm 6.02$ for $\mathrm{rFSH}$ vs. $9.77 \pm 5.53$ for HP-HMG; $p<0.01)$.

The economic evaluation presented in this study shows a similar cost per oocyte for $\mathrm{rFSH}$ and HMG, is the first costs analysis performed using Italian treatment costs. The minor cost difference found suggests that the higher unit cost of rFSH may be offset by a higher efficacy compared with HMG. Furthermore, clinicians should bear in mind that rFSH allows for more frozen embryo transfers than HMG, since it produces a greater number of oocytes; thus, it can be possible to reduce the number of ovarian stimulations, with a consequent minor overall treatment cost.

Additionally, Zhu et al. [31] found that embryo cryopreservation and subsequent transfer cycle under optimal conditions, as opposed to fresh transfer cycle, achieve improved synchrony between embryo and endometrial development, thereby enhancing the clinical outcome.

The safety and tolerability of rFSH have been extensively evaluated since it became available. The most obvious clinical safety advantages arise from the high purity of $\mathrm{rFSH}$; $\mathrm{rFSH}$ has been proven to have better overall tolerability than any previous FSH preparation [32]. In fact, filled by mass manufacturing process of follitropin $\alpha$ eliminates the intrinsic variability of the rat bioassay and ensures high batch-to-batch and vial-to-vial consistency of rFSH content. Furthermore, analytical assessment of commercially available $\mathrm{rFSH}$ pharmaceutical products has shown that follitropin $\alpha$ filled by mass is the most consistent $\mathrm{rFSH}$ in terms of protein content [33].

In contrast, since HMG preparations are directly extracted from human urine, the FSH activity in the preparations is highly variable between batches; the control of raw material of the individual contributors and the variation of purification processes are the major barriers in improving the quality of urinary preparations [34]. Systematic literature reviews provide an excellent method to address eventual deficiencies of individual trials by considering several clinical studies. However, differences in results among studies could exist and could depend on clinical trials with different design and clinical practice, rather than differences in participants and clinical settings. 
However, this review presents some limitations. Only peer-reviewed papers are included, hence there is the possibility of selection bias related to the publication source. In addition, even though the searches are done thoroughly through multiple major databases with cross-referencing, there is a possibility that some papers with pivotal findings for this issue have not been included in this current review. However, since no selection criteria on patients' characteristics, indications, or treatment protocols were applied, publications selected cover a wide range of interventions and medical settings, which are representative of the use of gonadotropins.

Using just the number of oocytes retrieved per cycle as the only outcome to evaluate the efficacy of rFSH and HMG could be a limitation of this analysis. Nevertheless, the oocytes yield per cycle could be considered a direct measure of gonadotropin stimulation and has been demonstrated to be highly correlated with live birth rate $[10,11]$. Furthermore, Stoop et al. [35] demonstrated that a higher number of oocytes reduces cancelation rates, reduces the risk for multiple pregnancies and may lead to future pregnancies.

Any oocyte retrieved, independently from its maturation stage, was included in this analysis, due to the absence of information in the majority of the studies. However, Mehri et al. [36] showed that only mature oocytes have an increased fertilization rate.

The authors applied the Italian acquisition costs for HPHMG to all HMG medications, whether highly purified or not. However, only a highly purified formulation is present in Italy. Furthermore, the cost of production related to a more sophisticated process could plausibly be more expensive, generating an underestimate of the costs associated with the HMG at a lower purification rate.

In conclusion, considering the number of oocytes retrieved as the best direct measure of efficient ovarian stimulation and considering the strong correlation between egg number and live birth, rFSH resulted to be more effective in comparison with HMG.

Despite a relatively high acquisition cost of $\mathrm{rFSH}$, the use of recombinant therapy for the treatment of infertility in an Italian perspective generates a cost per oocyte similar to the cost associated to HMG due to higher oocytes yield.

Conflict of interest This study was financially supported by Merck Serono $\mathrm{SpA}$, which was not responsible for creation of the study documents, the data analysis, data interpretation, or writing of the manuscript. SL is an employee of Merck Serono SpA. CR is an employee of CSD Medical Research Srl. PELS, CA, GC, CP, PLC and GPD have received honoraria by Merck Serono SpA. The authors report no other conflicts of interest in this work. All authors were responsible for data interpretation and reviewed and approved the final manuscript.
Open Access This article is distributed under the terms of the Creative Commons Attribution License which permits any use, distribution, and reproduction in any medium, provided the original author(s) and the source are credited.

\section{References}

1. Parhar IS (2002) Gonadotropin-releasing hormone: molecules and receptors. Elsevier, Amsterdam

2. Donini P, Montezemolo R (1949) Rassegna di Clinica, Terapia e Scienze Affini. A publ biol lab inst serono 48:3-28

3. Rosenberg E, Coleman J, Damani M, Garcia CR (1962) Clinical effect of post menopausal gonadotropin. J Clin Endocrinol Metab 23:181-189

4. Lunenfeld B (1963) Treatment of anovulation by human gonadotropins. J Int Fed Gyneacol Obstet 1:153

5. Eshkol A, Lunenfeld B (1967) Purification and separation of follicle stimulating hormone (FSH) and luteinizing hormone ( $\mathrm{LH})$ from human menopausal gonadotrophin (HMG) Part III. Acta Endocrinol 54:919

6. Hugues JN (1994) Metrodin HP: a new highly purified FSH. Contracept Fertil Sex 22(6):402-404

7. Lunenfeld B, Lunenfeld E (1997) Ovulation induction with human menopausal gonadotropin. In: Seibel MM (ed) Infertility: a comprehensive text, Norwalk, CT, Appleton \& Lange, pp 507-523

8. Hull M, Corrigan E, Piazzi A, Loumaye E (1994) Recombinant human luteinising hormone: an effective new gonadotropin preparation. Lancet 344:334-335

9. Loumaye E, Campbell R, Salat-Baroux J (1995) Human folliclestimulating hormone produced by recombinant DNA technology: a review for clinicians. Hum Reprod 1(2):188-199

10. Sunkara SK, Rittenberg V, Raine-Fenning N, Bhattacharya S, Zamora J, Coomarasamy A (2011) Association between the number of eggs and live birth in IVF treatment: an analysis of 400 135 treatment cycles. Hum Reprod 26(7):1768-1774

11. Stoop D, Ermini B, Polyzos NP, Haentjens P, De Vos M, Verheyen G, Devroey P (2012) Reproductive potential of a metaphase II oocyte retrieved after ovarian stimulation: an analysis of 23354 ICSI cycles. Hum Reprod 27(7):2030-2035

12. Jansen CA, van Os HC, Out HJ, Coelingh Bennink HJ (1998) A prospective randomized clinical trial comparing recombinant follicle stimulating hormone (Puregon) and human menopausal gonadotrophins (Humegon) in non-down-regulated in vitro fertilization patients. Hum Reprod 13(11):2995-2999

13. Gordon UD, Harrison RF, Fawzy M, Hennelly B, Gordon AC (2001) A randomized prospective assessor-blind evaluation of luteinizing hormone dosage and in vitro fertilization outcome. Fertil steril. 75(2):324-331

14. Ng EH, Lau EY, Yeung WS, Ho PC (2001) HMG is as good as recombinant human FSH in terms of oocyte and embryo quality: a prospective randomized trial. Hum Reprod 16(2):319-325

15. Strehler E, Abt M, El-Danasouri I, De Santo M, Sterzik K (2001) Impact of recombinant follicle-stimulating hormone and human menopausal gonadotropins on in vitro fertilization outcome. Fertil Steril 75(2):332-336

16. Westergaard LG, Erb K, Laursen SB, Rex S, Rasmussen PE (2001) Human menopausal gonadotropin versus recombinant follicle-stimulating hormone in normogonadotropic women downregulated with a gonadotropin-releasing hormone agonist who were undergoing IVF and ICSI: a prospective randomized study. Fertil Steril 76(3):543-549

17. Balasch J, Peñarrubia J, Fábregues F, Vidal E, Casamitjana R, Manau D, Carmona F, Creus M, Vanrell JA., "Ovarian responses 
to recombinant FSH or HMG in normogonadotrophic women following pituitary desensitization by a depot GnRH agonist for assisted reproduction," Reprod Biomed Online, vol. 7, no. 1, pp. 35-42, Jul-Aug 2003

18. Kilani Z, Dakkak A, Ghunaim S, Cognigni GE, Tabarelli C, Parmegiani L, Filicori M (2003) A prospective, randomized, controlled trial comparing highly purified hMG with recombinant FSH in women undergoing ICSI: ovarian response and clinical outcomes. Hum Reprod 18(6):1194-1199

19. Rashidi BH, Sarvi F, Tehrani ES, Zayeri F, Movahedin M, Khanafshar N (2005) The effect of HMG and recombinant human FSH on oocyte quality: a randomized single-blind clinical trial. Eur J Obstet Gynecol Reprod Biol 120(2):190-194

20. Andersen AN, Devroey P, Arce JC (2006) nical outcome following stimulation with highly purified $\mathrm{hMG}$ or recombinant FSH in patients undergoing IVF: a randomized assessor-blind controlled trial. Hum Reprod 21(12):3217-3227

21. Bosch E, Vidal C, Labarta E, Simon C, Remohi J, Pellicer A (2008) Highly purified hMG versus recombinant FSH in ovarian hyperstimulation with GnRH antagonists-a randomized study. Hum Reprod 23(10):2346-2351

22. Hompes PG, Broekmans FJ, Hoozemans DA, Schats R (2008) Effectiveness of highly purified human menopausal gonadotropin vs. recombinant follicle-stimulating hormone in first-cycle in vitro fertilization-intracytoplasmic sperm injection patients. Fertil Steril 89(6):1685-1693

23. Devroey P, Pellicer A, Nyboe Andersen A, Arce JC (2012) A randomized assessor-blind trial comparing highly purified hMG and recombinant FSH in a GnRH antagonist cycle with compulsory single-blastocyst transfer. Fertil Steril 97(3):561-571

24. Ye H, Huang G, Pei L, Zeng P, Luo X (2012) Outcome of in vitro fertilization following stimulation with highly purified hMG or recombinant FSH in downregulated women of advanced reproductive age: a prospective, randomized and controlled trial. Gynecol Endocrinol 28(7):540-544

25. Codifa Database. Informatore Farmaceutico online. http://www. codifa.it

26. van Wely M, Kwan I, Burt AL, Thomas J, Vail A, Van der Veen F, Al-Inany HG (2012) Recombinant versus urinary gonadotrophin for ovarian stimulation in assisted reproductive technology cycles. A Cochrane review. Hum Reprod Update 18(2):111

27. Jee BC, Suh CS, Kim YB, Kim SH, Moon SY (2010) Clinical efficacy of highly purified hMG versus recombinant FSH in IVF/ICSI cycles: a meta-analysis. Gynecol Obstet Invest 70(2):132-137
28. Lehert P, Schertz JC, Ezcurra D (2010) Recombinant human follicle-stimulating hormone produces more oocytes with a lower total dose per cycle in assisted reproductive technologies compared with highly purified human menopausal gonadotrophin: a meta-analysis. Reprod Biol Endocrinol 16(8):112

29. Wex J, Abou-Setta AM (2013) Economic evaluation of highly purified human menopausal gonadotropin versus recombinant human follicle-stimulating hormone in fresh and frozen in vitro fertilization/intracytoplasmic sperm-injection cycles in Sweden. Clinicoecon Outcomes Res 9(5):381-397

30. Trew GH, Brown AP, Gillard S, Blackmore S, Clewlow C, O'Donohoe P, Wasiak R (2013) In vitro fertilisation with recombinant follicle stimulating hormone requires less IU usage compared with highly purified human menopausal gonadotrophin: results from a European retrospective observational chart review. Reprod Biol Endocrinol 8(8):137

31. Zhu D, Zhang J, Cao S, Zhang J, Heng BC, Huang M, Ling X, Duan T, Tong GQ (2011) Vitrified-warmed blastocyst transfer cycles yield higher pregnancy and implantation rates compared with fresh blastocyst transfer cycles-time for a new embryo transfer strategy? Fertil Steril 95(5):1691-1695

32. Devroey P, Ubaldi F, Smitz J, Van Steirteghem A (1994) Recombinant follicle stimulating hormone. Assist Reprod Rev 4(1):2-9

33. Bassett R, De Bellis C, Chiacchiarini L, Mendola D, Micangeli E, Minari K, Grimaldi L, Mancinelli M, Mastrangeli R, Bucci $\mathrm{R}$ (2005) Comparative characterisation of a commercial human chorionic gonadotrophin extracted from human urine with a commercial recombinant human chorionic gonadotrophin. Curr Med Res Opin 21(12):1969-1976

34. Bassett R, Lispi M, Ceccarelli D, Grimaldi L, Mancinelli M, Martelli F, Van Dorsselaer A (2009) Analytical identification of additional impurities in urinary-derived gonadotrophins. Reprod Biomed Online 19(3):300-313

35. Stoop D, Van Landuyt L, Paquay R, Fatemi H, Blockeel C, De Vos M, Camus M, Van den Abbeel E, Devroey P (2010) Offering excess oocyte aspiration and vitrification to patients undergoing stimulated artificial insemination cycles can reduce the multiple pregnancy risk and accumulate oocytes for later use. Hum Reprod 25(5):1213-1218

36. Mehri S, Levi Setti PE, Greco K, Sakkas D, Martinez G, Patrizio $P$ (2014) Correlation between follicular diameters and flushing versus no flushing on oocyte maturity, fertilization rate and embryo quality. J Assist Reprod Genet 31(1):73-77 